\title{
Risk factors for post-COVID-19 condition in previously hospitalised children using the ISARIC Global follow-up protocol: a prospective cohort study
}

\author{
Ismail M. Osmanov ${ }^{1,2,29}$, Ekaterina Spiridonova ${ }^{3,29}$, Polina Bobkova ${ }^{3,29}$, Aysylu Gamirova ${ }^{3,29}$, \\ Anastasia Shikhaleva $^{3,29}$, Margarita Andreeva ${ }^{3,29}$, Oleg Blyuss ${ }^{3,4,29}$, Yasmin El-Taravi ${ }^{3}$, \\ Audrey DunnGalvin ${ }^{3,5}$, Pasquale Comberiati ${ }^{6}$, Diego G. Peroni $\mathbb{1}^{6}$, Christian Apfelbacher ${ }^{7}$, Jon Genuneit $\mathbb{C}^{8}$, \\ Lyudmila Mazankova $\mathbb{1}^{9}$, Alexandra Miroshina ${ }^{1}$, Evgeniya Chistyakova ${ }^{10}$, Elmira Samitova ${ }^{1,9}$, \\ Svetlana Borzakova ${ }^{2,11}$, Elena Bondarenko ${ }^{3}$, Anatoliy A. Korsunskiy ${ }^{3}$, Irina Konova $\mathbb{1}^{1}$, \\ Sarah Wulf Hanson ${ }^{12}$, Gail Carson ${ }^{13}$, Louise Sigfrid $\mathbb{1}^{13}$, Janet T. Scott ${ }^{14}$, Matthew Greenhawt ${ }^{15}$, \\ Elizabeth A. Whittaker ${ }^{16}$, Elena Garralda ${ }^{17}$, Olivia V. Swann ${ }^{18,19}$, Danilo Buonsenso $\mathbb{1}^{20,21,22}$, \\ Dasha E. Nicholls ${ }^{17}$, Frances Simpson ${ }^{23}$, Christina Jones $\mathbb{1}^{24}$, Malcolm G. Semple $\mathbb{1}^{25,26}$, John 0 . Warner ${ }^{27}$, \\ Theo Vos $^{12}$, Piero Olliaro ${ }^{13}$, Daniel Munblit $\mathbb{1}^{3,27,28,29}$ and the Sechenov StopCOVID Research Team ${ }^{30}$
}

\begin{abstract}
${ }^{1}$ Z.A. Bashlyaeva Children's Municipal Clinical Hospital, Moscow, Russia. ${ }^{2}$ Pirogov Russian National Research Medical University, Moscow, Russia. ${ }^{3}$ Dept of Paediatrics and Paediatric Infectious Diseases, Institute of Child's Health, Sechenov First Moscow State Medical University (Sechenov University), Moscow, Russia. ${ }^{4}$ School of Physics, Astronomy and Mathematics, University of Hertfordshire, Hatfield, UK. ${ }^{5}$ School of Applied Psychology, University College Cork, Cork City, Ireland. ${ }^{6}$ Dept of Clinical and Experimental Medicine, Section of Pediatrics, University of Pisa, Pisa, Italy. ${ }^{7}$ Institute of Social Medicine and Health Systems Research, Faculty of Medicine, Otto von Guericke University Magdeburg, Magdeburg, Germany. ${ }^{8}$ Pediatric Epidemiology, Dept of Pediatrics, Medical Faculty, Leipzig University, Leipzig, Germany. ${ }^{9}$ Russian Medical Academy of Continuous Professional Education of the Ministry of Healthcare of the Russian Federation, Moscow, Russia. ${ }^{10}$ Dept of Paediatrics and Paediatric Rheumatology, Institute of Child's Health, Sechenov First Moscow State Medical University (Sechenov University), Moscow, Russia. ${ }^{11}$ Research Institute for Healthcare Organization and Medical Management of Moscow Healthcare Dept, Moscow, Russia. ${ }^{12}$ Institute for Health Metrics and Evaluation, University of Washington, Seattle, WA, USA. ${ }^{13}$ ISARIC Global Support Centre, Nuffield Dept of Medicine, University of Oxford, Oxford, UK. ${ }^{14}$ MRC-University of Glasgow Centre for Virus Research, Glasgow, UK. ${ }^{15}$ Dept of Pediatrics, Section of Allergy/Immunology, Children's Hospital Colorado, University of Colorado School of Medicine, Aurora, CO, USA. ${ }^{16}$ Paediatric Infectious Diseases, Imperial College Healthcare NHS Trust, London, UK. ${ }^{17}$ Division of Psychiatry, Imperial College London, London, UK. ${ }^{18}$ Dept of Child Life and Health, University of Edinburgh, Edinburgh, UK. ${ }^{19}$ Paediatric Infectious Diseases, Royal Hospital for Children, Glasgow, UK. ${ }^{20}$ Dept of Woman and Child Health and Public Health, Fondazione Policlinico Universitario A. Gemelli IRCCS, Rome, Italy. ${ }^{21}$ Dipartimento di Scienze Biotecnologiche di Base, Cliniche Intensivologiche e Perioperatorie, Università Cattolica del Sacro Cuore, Rome, Italy. ${ }^{22}$ Center for Global Health Research and Studies, Università Cattolica del Sacro Cuore, Rome, Italy. ${ }^{23}$ Coventry University, Coventry, UK. ${ }^{24}$ School of Psychology, Faculty of Health and Medical Sciences, University of Surrey, Guildford, UK. ${ }^{25}$ Health Protection Research Unit in Emerging and Zoonotic Infections, Institute of Infection, Veterinary and Ecological Sciences, Faculty of Health and Life Sciences, University of Liverpool, Liverpool, UK. ${ }^{26}$ Dept of Respiratory Medicine, Alder Hey Children's Hospital, Liverpool, UK. ${ }^{27}$ Inflammation, Repair and Development Section, National Heart and Lung Institute, Faculty of Medicine, Imperial College London, London, UK. ${ }^{28}$ Research and Clinical Center for Neuropsychiatry, Moscow, Russia. ${ }^{29}$ These authors contributed equally to this article. ${ }^{30} \mathrm{~A}$ list of members of the Sechenov StopCOVID Research Team can be found in the Acknowledgements section.
\end{abstract}

Corresponding author: Daniel Munblit (daniel.munblit08@imperial.ac.uk)

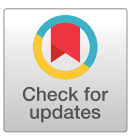

Shareable abstract (@ERSpublications)

A quarter of children experienced persistent symptoms months after COVID-19 infection, with almost one in 10 experiencing multisystem involvement. Older age and allergic diseases were associated with higher risk of persistent symptoms at follow-up. https://bit.ly/3vqeEmZ

Cite this article as: Osmanov IM, Spiridonova E, Bobkova P, et al. Risk factors for post-COVID-19 condition in previously hospitalised children using the ISARIC Global follow-up protocol: a prospective cohort study. Eur Respir J 2022; 59: 2101341 [DOI: 10.1183/13993003.01341-2021]. 
This version is distributed under the terms of the Creative Commons Attribution Non-Commercial Licence 4.0. For commercial reproduction rights and permissions contact permissions@ersnet.org

This article has an editorial commentary: https://doi.org/10.1183/ $13993003.02245-2021$

Received: 10 May 2021 Accepted: 9 June 2021
Methods This is a prospective cohort study of children ( $\leqslant 18$ years old) admitted to hospital with confirmed COVID-19. Children admitted between 2 April 2020 and 26 August 2020 were included. Telephone interviews used the International Severe Acute Respiratory and Emerging Infection Consortium (ISARIC) COVID-19 Health and Wellbeing Follow-up Survey for Children. Persistent symptoms (>5 months) were further categorised by system(s) involved.

Results 518 out of 853 (61\%) eligible children were available for the follow-up assessment and included in the study. Median (interquartile range (IQR)) age was 10.4 (3-15.2) years and 270 (52.1\%) were girls. Median (IQR) follow-up since hospital discharge was 256 (223-271) days. At the time of the follow-up interview 126 (24.3\%) participants reported persistent symptoms, among which fatigue (53, 10.7\%), sleep disturbance (36, 6.9\%) and sensory problems (29, 5.6\%) were the most common. Multiple symptoms were experienced by 44 (8.4\%) participants. Risk factors for persistent symptoms were: older age "6-11 years" (OR 2.74, 95\% CI 1.37-5.75) and “12-18 years” (OR 2.68, 95\% CI 1.41-5.4), and a history of allergic diseases (OR 1.67, 95\% CI 1.04-2.67).

Conclusions A quarter of children experienced persistent symptoms months after hospitalisation with acute COVID-19 infection, with almost one in 10 experiencing multisystem involvement. Older age and allergic diseases were associated with higher risk of persistent symptoms at follow-up.

\section{Introduction}

Emerging data suggest that a substantial proportion of people experience ongoing symptoms including fatigue and muscle weakness, breathlessness, and neurological problems more than 6 months after the acute phase of coronavirus disease 2019 (COVID-19) [1, 2]. This phenomenon is commonly referred to as "long COVID", a term defined by patient groups, and also known as post-COVID-19 syndrome, the post-COVID-19 condition [3] or "COVID long-haulers" [4, 5]. Recent population data from the UK reported that the highest prevalence of long COVID after 12 weeks was among those aged 25-34 years (18.2\%) and lowest in those aged 2-11 years (7.4\%) [6].

Evidence on post-acute COVID-19 condition and long-term outcomes in children is still limited to small studies, with more than half having at least one persisting symptom 4 months after COVID-19 infection [7]. However, a recent publication from Australia by SAY et al. [8] suggested that only 8\% of children aged 0-19 years (median 3 years) had ongoing symptoms 3-6 months after predominantly mild COVID-19 infection. The limitation of the study as acknowledged by the authors was the low age range. This mandates the inclusion of larger numbers, particularly of older children, in future studies [8].

There is a need to assess the long-term consequences of COVID-19 in paediatric populations [9], to inform clinicians, researchers and public health experts, to address the impacts of this condition on those affected and their families, and to inform discussions on vaccination of children. This cohort study aimed to investigate the incidence of and risk factors for long-term COVID-19 outcomes in children post-hospital discharge. We used the standardised follow-up data collection protocol developed by the International Severe Acute Respiratory and Emerging Infection Consortium (ISARIC) Global Paediatric COVID-19 Follow-up Working Group [10].

\section{Methods}

\section{Study design, setting and participants}

This is a prospective cohort study of children ( $\leqslant 18$ years old) admitted with suspected or confirmed COVID-19 to Z.A. Bashlyaeva Children’s Municipal Clinical Hospital in Moscow, Russia. This large tertiary university hospital can accommodate up to 980 children at a time and served as the primary COVID-19 hospital for children residing in Moscow city. Children admitted to the hospital during the first wave of the pandemic, between 2 April 2020 and 26 August 2020, with reverse transcriptase PCR-confirmed severe acute respiratory syndrome coronavirus 2 (SARS-CoV-2) infection were included. The parents of these children were contacted between 31 January 2021 and 27 February 2021 to complete a follow-up survey for this study.

The acute-phase dataset included demographics, symptoms, comorbidities, chest computed tomography, supportive care and clinical outcomes at discharge. This study was approved by the Moscow City Independent Ethics Committee (abbreviate 1, protocol number 74). Parental consent was sought during hospital admission and consent for the follow-up interview was sought via verbal confirmation during telephone interview.

Interviews were undertaken by a team of medical students with experience gained in previous COVID-19 research [2,11] who underwent standardised training in telephone assessment, REDCap data entry and data security. Assessments were conducted via interviews with the parents/carers. Nonresponders were 
contacted by telephone three times before considering them lost to follow-up. Information about the current condition and persisting symptoms was collected using version 1.0 of the ISARIC COVID-19 Health and Wellbeing Follow-Up Survey for Children, to assess patients' physical and psychosocial wellbeing and behaviour, with local adaptations (additional questions related to the presence and duration of signs/symptoms were included), translated into Russian. The protocol was registered at The Open Science Framework [12]. The follow-up survey documented data on demographics, parental perception of changes in their child's emotional and behavioural status (including reasons for change: COVID-19, pandemic or both), previous vaccination history, hospital stay and readmissions, mortality (after the initial index event), history of newly developed symptoms between discharge and the follow-up assessment, including symptom onset and duration, and overall health condition compared with prior to the child's COVID-19 onset (supplementary material). To assess the prevalence of symptoms over time parents were asked the following questions: "Within the last seven days, has your child had any of these symptoms, which were NOT present prior to their Covid-19 illness? (If yes, please indicate below and the duration of the symptom/s)" and "Please report any symptoms that have been bothering your child since discharge that are not present today. Please specify the time of onset and duration of these symptoms”.

\section{Data management}

REDCap electronic data capture tools (www.project-redcap.org) hosted at Sechenov University (Moscow, Russia) and Microsoft Excel (Microsoft, Redmond, WA, USA) were used for data collection, storage and management $[13,14]$. The baseline characteristics, including demographics, symptoms on admission and comorbidities, were extracted from electronic medical records and entered into REDCap.

\section{Exposure and outcome variables}

For the purposes of this study, we defined "persistent symptoms" as symptoms present at the time of the follow-up interview and lasting for $>5$ months. These were subcategorised into respiratory, neurological, sensory, sleep, gastrointestinal, dermatological, cardiovascular, fatigue and musculoskeletal (supplementary table S1), as informed by previously published literature [15, 16] and ISARIC Global Paediatric COVID-19 Follow-up Working Group discussions.

Allergic diseases were defined as the presence of any of the following: asthma, allergic rhinitis, eczema or food allergy. Participants' age categories were based on Eunice Kennedy Shriver National Institute of Child Health and Human Development Pediatric Terminology [17]. Severe disease was defined as having received noninvasive ventilation, invasive ventilation or admission to the paediatric intensive care unit (PICU) during hospital admission.

Health status before COVID-19 and at the time of the interview was assessed using a 0-100 wellness scale [18], where 0 was the worst possible health and 100 was the best possible health.

\section{Statistical analysis}

Descriptive statistics were calculated for baseline characteristics. Continuous variables were summarised as median (interquartile range (IQR)) and categorical variables as frequency (percentage). The Chi-squared test or Fisher's exact test was used for testing hypotheses on differences in proportions between groups. The Wilcoxon rank-sum test was used for testing hypotheses on differences between groups.

We performed multivariable logistic regression to investigate associations of demographic characteristics, comorbidities (limited to those reported in $\geqslant 5 \%$ of participants), presence of pneumonia and severity of COVID-19 during acute infection with persistent symptom categories present at the time of the follow-up interview. We included all participants for whom the variables of interest were available in the final analysis, without imputing missing data. The differing denominators used indicate missing data. Odds ratios were calculated together with $95 \%$ confidence intervals.

UpSet plots were used to present the coexistence of persistent symptom categories. Two-sided p-values were reported for all statistical tests; a p-value $<0.05$ was considered to be statistically significant. Statistical analysis was performed using R version 3.5.1 (https://cran.r-project.org). Packages used included dplyr, lubridate, ggplots2, plotrix and UpSetR.

\section{Patient and public involvement}

The survey was developed by the ISARIC Global Paediatric COVID-19 Follow-up Working Group and informed by a wide range of global stakeholders with expertise in infectious diseases, critical care, paediatrics, epidemiology, allergy/immunology, respiratory medicine, psychiatry, psychology and 
methodology, and patient representatives. The survey was distributed to the members of the patient group and suggestions from parents/carers were implemented.

\section{Results}

All 853 children hospitalised with suspected COVID-19 between 2 April 2020 and 26 August 2020 were discharged alive (figure 1). Of 836 patients with accurate contact information, parents of 518 PCR-positive children agreed to be interviewed (response rate 62\%) and were included in the analysis.

Median (IQR) age was 10.4 (3-15.2) years (range 2 days-18 years) and 272 (52.1\%) were girls. Median (IQR) follow-up time since hospital admission was 268 (233-284) days. Children had a median (IQR) of 8 (4-9) years of formal school education and a median (IQR) of 4 (3-5) family members were residing in the household (table 1).

The most common pre-existing comorbidity in this cohort was food allergy (13\% (67/514)), followed by allergic rhinitis and asthma (9.7\% (50/514)), gastrointestinal problems (9.3\% (48/514)), eczema (8.8\% (45/ $514)$ ), and neurological conditions (8.8\% (45/514)). Parents of 55.3\% (284/514) of children did not report any comorbidities. Fever (83.6\% (427/511)), cough (55.7\% (284/510)), rhinorrhoea (54.3\% (278/512)) and fatigue (38.9\% (197/506)) were the most common presenting symptoms at the time of hospital admission (supplementary table S2). 37.3\% (192/515) of patients had pneumonia during the hospital stay; 2.7\% (14/ 515 ) had severe disease, which required noninvasive ventilation/invasive ventilation or admission to the PICU. Treatments received during hospital admission are presented in supplementary table S3.

At the time of the follow-up interview, parents of $24.7 \%$ (128) of children reported at least one persistent symptom, with fatigue $10.6 \%$ (53/496), insomnia 5.19\% (26/501), disturbed smell 4.7\% (22/467) and headache $3.5 \%$ (17/486) being the most common. Detailed information on symptoms and duration is presented in supplementary table S4.

The prevalence of the symptoms present at the time of discharge declined over time (figure 2). The number of children with fatigue fell from $15.8 \%(82 / 518)$ at the time of discharge to $8.8 \%(45 / 513)$ 6-7 months later, altered sense of smell from $8.7 \%$ (45/518) to $4.7 \%(24 / 514)$, sleep disturbance from $7.5 \%(39 / 518)$ to $5.8 \%(30 / 515)$, altered sense of taste from $5.6 \%(29 / 518)$ to $3.1 \%(16 / 515)$, headache from $4.6 \%(24 / 518)$ to $3.5 \%(18 / 517)$ and breathing difficulties from $3.9 \%(20 / 518)$ to $1 \%(5 / 517)$.

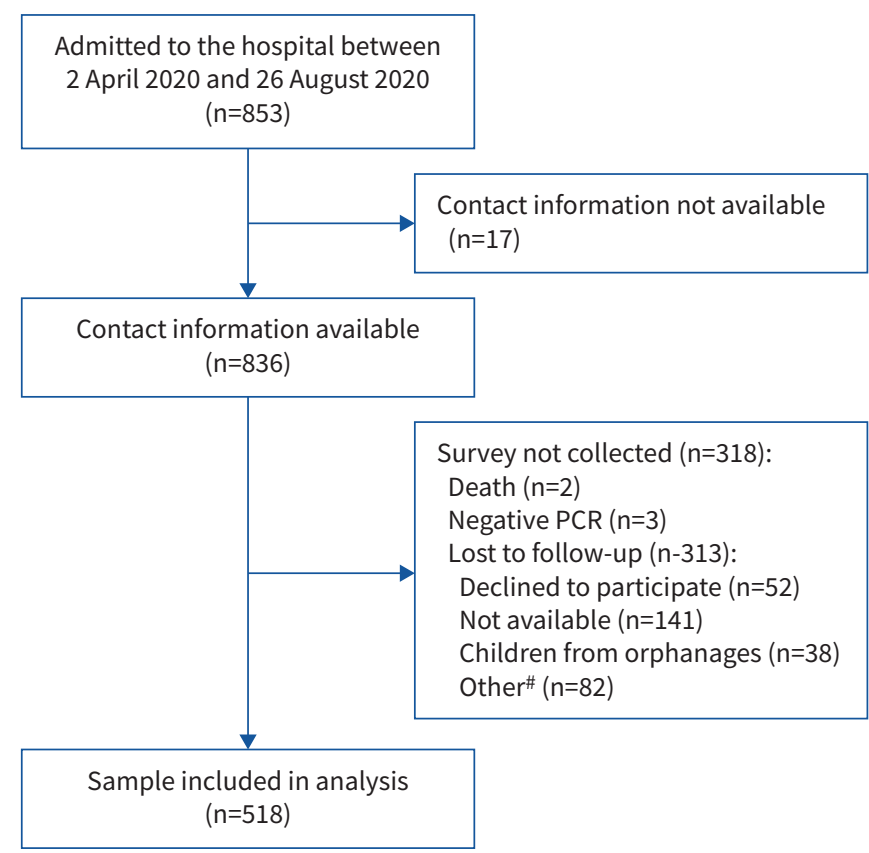

FIGURE 1 Flow diagram of patients with COVID-19 admitted to Z.A. Bashlyaeva Children's Municipal Clinical Hospital between 2 April 2020 and 26 August 2020. ": relatives unable to describe the child's health; relatives not willing to refer interviewers to the child's parents/carers; inability to speak Russian. 
TABLE 1 Demographic characteristics of patients with COVID-19 admitted to Z.A. Bashlyaeva Children's Municipal Clinical Hospital

\begin{tabular}{|c|c|}
\hline Female & $270 / 518(52.1)$ \\
\hline Age at hospital admission, years & $10.4(3-15.2)$ \\
\hline \multicolumn{2}{|l|}{ Age group } \\
\hline$<2$ years & $105 / 518(20.3)$ \\
\hline $2-5$ years & $80 / 518(15.4)$ \\
\hline $6-11$ years & $113 / 518(21.8)$ \\
\hline $12-18$ years & $220 / 518(42.5)$ \\
\hline Time from discharge to follow-up, days & $256(223-271)$ \\
\hline Length of hospital admission, days & $10(7-14)$ \\
\hline Formal school education, years & $8(4-9)$ \\
\hline Members in household, $\mathrm{n}$ & $4(3-5)$ \\
\hline Pneumonia during hospitalisation & $192 / 515(37.3)$ \\
\hline Severe disease (noninvasive ventilation/invasive ventilation or PICU) & $14 / 515(2.7)$ \\
\hline \multicolumn{2}{|l|}{ Comorbidities } \\
\hline Neurological conditions & $45 / 514(8.8)$ \\
\hline Neurological disorders & $43 / 514(8.4)$ \\
\hline Neurodisability & $11 / 514(2.1)$ \\
\hline Heart diseases & $21 / 514(4.1)$ \\
\hline Haematological conditions & 10/514 (1.9) \\
\hline Tuberculosis & 9/514 (1.8) \\
\hline Respiratory diseases (not including asthma) & $16 / 514(3.1)$ \\
\hline Allergic diseases & $121 / 514(23.5)$ \\
\hline Food allergy & $67 / 514(13)$ \\
\hline Allergic rhinitis & $46 / 514(8.9)$ \\
\hline Eczema & $45 / 514(8.8)$ \\
\hline Asthma (doctor-diagnosed) & $12 / 514(2.3)$ \\
\hline Other skin problems (not including eczema) & $8 / 514(1.6)$ \\
\hline Gastrointestinal problems & $48 / 514(9.3)$ \\
\hline Oncological conditions & $3 / 514(0.6)$ \\
\hline Immune system diseases & $6 / 514(1.2)$ \\
\hline Genetic conditions & $6 / 514(1.2)$ \\
\hline Diabetes $^{\#}$ & $3 / 514(0.6)$ \\
\hline Other endocrine illness (not including diabetes) & $12 / 514(2.3)$ \\
\hline Renal/kidney problems & $18 / 514(3.5)$ \\
\hline Excessive weight and obesity & $25 / 514(4.9)$ \\
\hline Malnutrition & $10 / 514(1.9)$ \\
\hline Rheumatological conditions & $4 / 514(0.8)$ \\
\hline Depression & $4 / 514(0.8)$ \\
\hline Anxiety & $5 / 514(1)$ \\
\hline HIV & $0 / 514(0)$ \\
\hline No comorbidities & $284 / 514(55.3)$ \\
\hline One comorbidity & $141 / 514(27.4)$ \\
\hline Two comorbidities or more & $89 / 514(17.3)$ \\
\hline
\end{tabular}

The prevalence of the most common symptoms, including symptoms that developed some time after discharge, is shown in supplementary figure S1.

With regard to persistent symptom categories (supplementary table S1), fatigue was the most commonly reported in 10.6\% (53/498) of patients at the time of assessment, followed by sleep disturbance 7.2\% (36/ 501), sensory 6.2\% (29/467), gastrointestinal 4.4\% (22/499) and dermatological 3.6\% (18/496) problems. A smaller number of patients experienced neurological 3\% (14/465), respiratory 2.5\% (12/489), cardiovascular 1.9\% (9/470) and musculoskeletal 1.8\% (9/489) problems long-term.

A total of $8.5 \%$ (44) of participants reported persistent symptoms from more than one category at the time of the follow-up assessment. The most commonly co-occurring categories were fatigue and sleep problems in $1.9 \%$ (10) of children, and fatigue and sensory problems were present in $1.5 \%$ (8) of children. $2.7 \%$ (14) of children had persistent symptoms from three or more different categories. Coexistence of persistent symptom categories at the time of follow-up is presented in the UpSet plot in figure 3. 


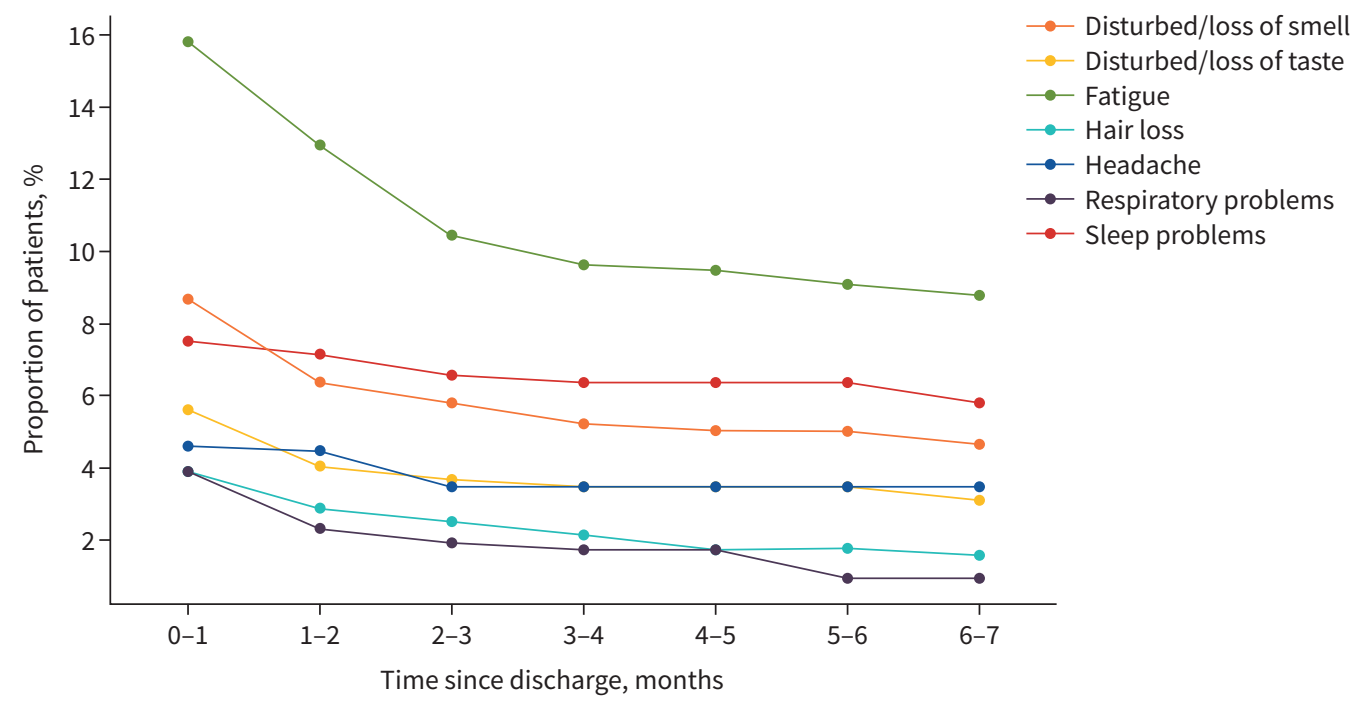

FIGURE 2 Duration of the most common symptoms (post-discharge) in children who experienced symptoms at the time of discharge. The calculations are based on responses to the questions: "Within the last seven days, has your child had any of these symptoms, which were NOT present prior to their Covid-19 illness? (If yes, please indicate below and the duration of the symptom/s)" and "Please report any symptoms that have been bothering your child since discharge that are not present today. Please specify the time of onset and duration of these symptoms".

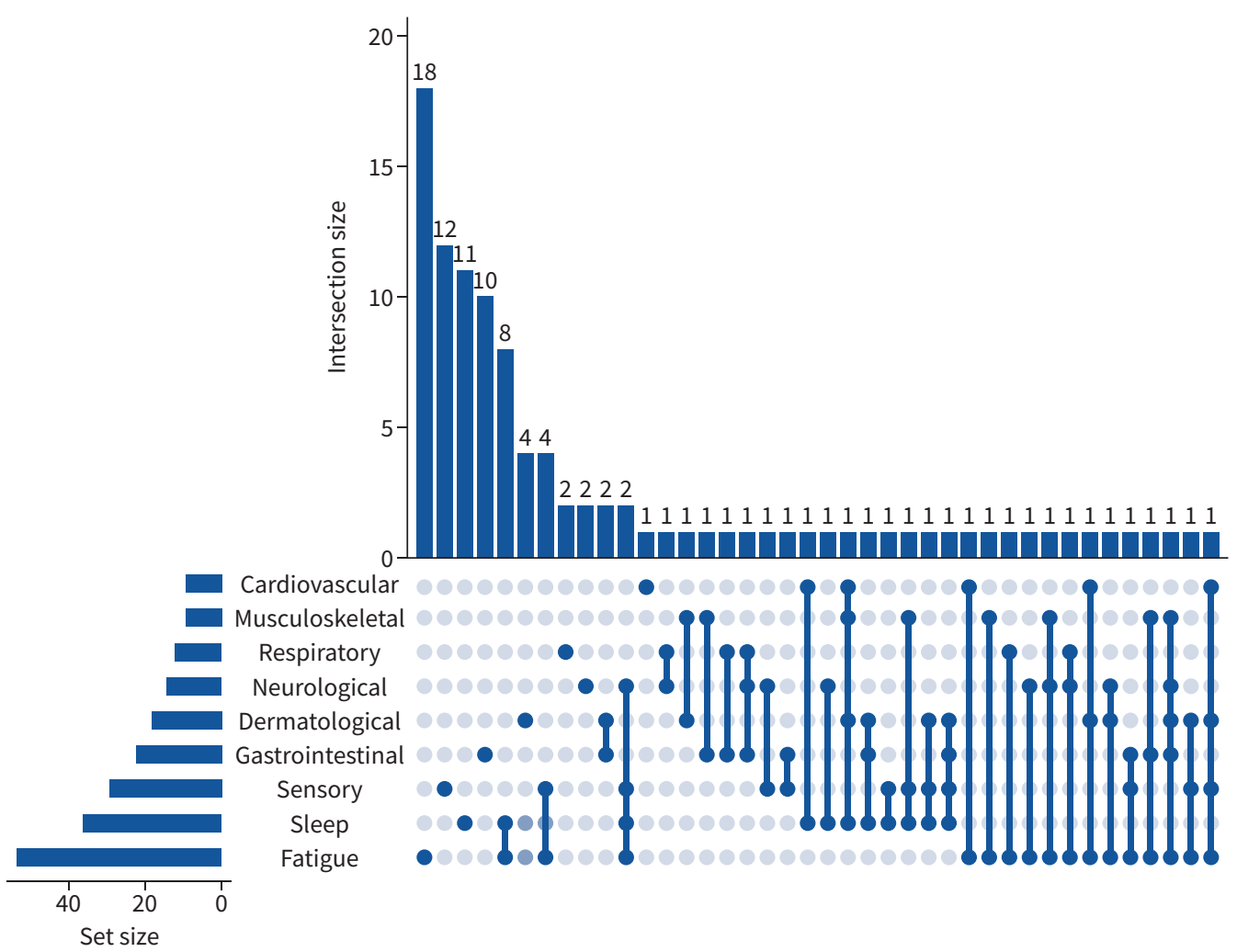

FIGURE 3 UpSet plot representing the coexistence of persistent symptom (present at the time of follow-up interview and lasting for $>5$ months) categories at follow-up assessment. The values represent the number of individuals experiencing a persistent symptom category or combination of categories. Dark blue lines link multiple symptoms indicated by dark blue circles. 
a)

Age $<2$ years

Age 2-5 years

Age 6-11 years

Age $12-18$ years

Female

Neurological conditions

Allergic diseases

Gastrointestinal problems

Excessive weight and obesity

Pneumonia during COVID-19

Severe acute COVID-19
Odds ratio $(95 \% \mathrm{Cl})$

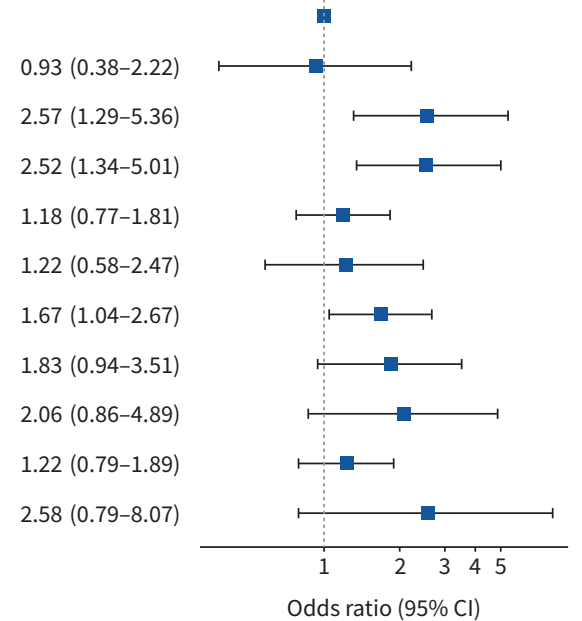

b)

Age $<2$ years

Age 2-5 years

Age 6-11 years

Age $12-18$ years

Female

Neurological conditions

Allergic diseases

Gastrointestinal problems

Excessive weight and obesity

Pneumonia during COVID-19

Severe acute COVID-19
Odds ratio $(95 \% \mathrm{Cl})$

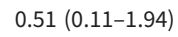

$2.49(1.02-6.72)$

$3.18(1.43-8.11)$

$1.06(0.63-1.78)$

$1.75(0.75-3.85)$

$1.58(0.88-2.76)$

$1.17(0.51-2.52)$

$2.50(0.97-6.08)$

0.99 (0.57-1.69)

$2.19(0.45-8.11)$

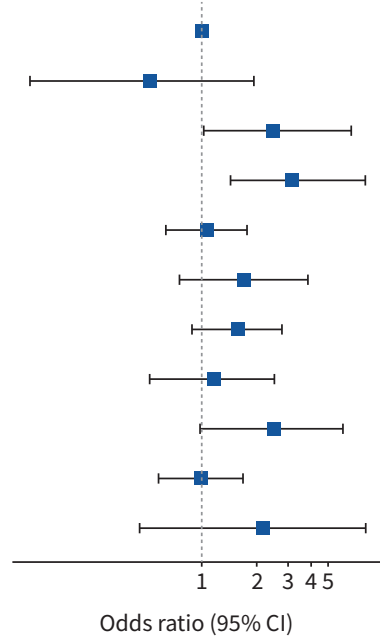

FIGURE 4 Multivariable logistic regression model to identify pre-existing risk factors for long COVID. Odds ratios (with 95\% confidence intervals) for the presence of a) any category of persistent symptoms $(n=127)$ at the time of follow-up and b) two or more coexisting categories of persistent symptoms $(n=73)$ at the time of follow-up. Neurological conditions and allergic diseases are specified in table 1 . Odds ratios are plotted on a log scale.

The scores on the wellness scale for children with one or two or more persistent symptoms significantly declined when compared with before COVID-19 onset, from 90 (80-100) to 82.5 (70-93.8) and from 90 (80-95) to 70 (60-80) ( $\mathrm{p}<0.001$ for all comparisons), respectively. Children who did not experience any persistent symptoms did not report any significant changes in wellness when asked to compare with how they felt before their acute COVID-19 illness. We also assessed emotional difficulties, social relationships and activity levels in children (supplementary tables S4 and S5). Parents related the following changes to COVID-19 illness and not to the pandemic in general: less eating in 4.5\% (23/512) of children, less sleeping in 3.5\% (18/511) and more sleeping in 2\% (10/511), reduced physical activity in 4.7\% (24/512), and child becoming less emotional in $4.3 \%$ (22/511). In contrast, parents attributed changes to social activities to the pandemic in general rather than to COVID-19 illness: 12\% (58/485) of children were spending less time with their friends in person, while 13\% (61/470) were spending more time with friends remotely, with less than $1 \%$ of parents attributing these changes to COVID-19 illness. 23\% (110/478) of children were spending more time watching television, playing video/computer games or using social media for educational purposes, with $92.9 \%$ of parents associating these changes with the pandemic in general rather than COVID-19 illness.

In multivariable regression analysis, older age was associated with persistent symptoms (figure 4a). When compared with children $<2$ years of age, those aged 6-11 years had OR 2.57 (95\% CI 1.29-5.36) for persistent symptoms and those aged 12-18 years had OR 2.52 (95\% CI 1.34-5.01). Another predictor associated with persistent symptoms was allergic diseases (OR 1.67, 95\% CI 1.04-2.67). Similar patterns were seen for children with coexistence of persistent symptoms from two or more categories: 6-11 years of age OR 2.49 (95\% CI 1.02-6.72) and 12-18 years of age OR 3.18 (95\% CI 1.43-8.11), both versus $<2$ years of age (figure $4 \mathrm{~b}$ ).

We ran an additional regression analyses using "age” as a continuous variable which gave a similar result (supplementary figure S2). When subgroup analyses were performed in the age group $\geqslant 6$ years, severe acute COVID-19 was associated with persistent symptoms (OR 6.14, 95\% CI 1.27-43.94) and excessive weight and obesity with coexistence of persistent symptoms from two or more categories (OR 2.89, 95\% CI 1.12-7.15) (supplementary figure S3).

\section{Discussion}

To the best our knowledge, this is the largest prospective paediatric cohort study with the longest follow-up assessing symptom prevalence and duration of long COVID in children and adolescents with laboratory-confirmed SARS-CoV-2 infection post-hospital discharge. We found that a quarter of children and adolescents had persistent symptoms at the time of follow-up, with fatigue, sleep disturbance and 
sensory problems being the most common. Almost one in 10 reported multisystem impacts with two or more categories of persistent symptoms at the time of follow-up. Children in mid-childhood and adolescence (aged 6-18 years) were at higher risk of persistent symptoms at the time of follow-up. Although prevalence of symptoms declined over time, a substantial proportion experienced problems many months after discharge.

Although many children experienced symptoms such as fatigue, disturbed smell and taste, sleep and respiratory problems, hair loss, and headaches at the time of hospital discharge, we witnessed a steady decline in symptom prevalence over time. This was particularly evident for fatigue and smell disturbance. Prevalence of some symptoms such as headache and sleep problems declined slower, which may be driven by psychological mechanisms rather than pathophysiological virus infection effects [19]. A limitation of these findings is that symptom onset and duration was recalled at the single follow-up interview in our study; this may be overcome with repeated follow-ups at appropriate intervals to limit potential recall imprecision. There are very few studies assessing long COVID in children and adolescents; a previous smaller study from Italy found similar persisting symptoms during a shorter follow-up [7]. In line with our results, previous research demonstrated symptoms fading over time in adults [15]; however, data are still limited as most of the published cohort studies do not measure the duration of symptoms, but rather assess their presence at a single follow-up.

We found that almost one in 10 children had multisystem impacts with two or more categories of persistent symptoms present at the time of follow-up. Similar numbers were previously reported in the Russian adult population [2] and patients with clusters of different symptoms were described in the UK [20]. Patients with multisystem involvement will represent the primary target for the development of future research and intervention strategies.

Age was significantly associated with persistent symptom presence at the time of follow-up, with children aged $\geqslant 6$ years being at higher risk. To the best of our knowledge, risk factors for long COVID in children have not been investigated in previous studies, so we may draw comparisons with the data from adult cohorts only. Previous data suggest that long COVID is prevalent in adults [1, 2, 20-23] and that age is associated with a higher risk of long COVID [20, 22]. An Australian follow-up study of 151 children (median 3 years) who had predominantly mild acute COVID-19 found only 8\% with ongoing long COVID symptoms [8]. As acknowledged by the authors of that study, low median age may be the main reason for the low long COVID prevalence and our study substantiates this. We also found that in children aged $\geqslant 6$ years, severe acute COVID-19 was associated with persistent symptoms and excessive weight and obesity with multisystem involvement, but confidence intervals were wide and these findings require confirmation on a larger sample size to make any firm conclusions.

We found that allergic diseases in children were also associated with a higher risk of long COVID. This is in agreement with adult studies from Russia [2] and the UK [20] reporting asthma to be associated with the development of long COVID. Recent data suggested that COVID-19 consequences may be linked with mast cell activation syndrome [24] and the T-helper type 2-biased immunological response in children with allergic diseases may be responsible for an increased risk of long-term consequences from the infection. This highlights the importance of further research on potential underlying immunological and autoimmune mechanisms of long COVID [25].

Apart from physical symptoms, we also assessed emotional and behavioural changes. Although most parents reported no changes, one in 20 parents noticed changes in their children that they attributed to COVID-19 illness rather than the general situation during the pandemic. These included changes in eating, sleeping, emotional wellbeing and physical activities. Over one in 10 parents noted that their children were spending less time in face-to-face communication and more time interacting with their friends remotely, and spending time online for both educational and noneducational purposes. These changes were largely attributed to the general situation during the pandemic rather than to COVID-19 illness. The "lockdown" measures were implemented in Moscow in the middle of March and lasted until June 2020. Restrictions included self-isolation, closure of public places, including schools/universities, social distancing, etc. The pandemic resulted in an increase in anxiety levels among the population, which was associated with increased media consumption [26]. The effect of the pandemic, illness or both should be further studied in future research.

A major strength of this study is that it was based on the ISARIC COVID-19 Health and Wellbeing Follow-Up Survey for Children, which will assist with data harmonisation and comparison with other international studies in the future. Another strength is the large sample size of confirmed 
SARS-CoV-2-infected children and this cohort has the longest follow-up assessment of hospitalised children to date. Stratification to determine if the symptoms were persistent following COVID-19 and assessment of trends over time were other novel aspects of the study. At the same time, this cohort study has several limitations. First, the study population only included patients within Moscow, although regional clustering is common to many cohort studies published during the COVID-19 pandemic. Second, it only included hospitalised children, not representative of the paediatric population. Third, we did not have a control group of previously hospitalised children not experiencing COVID-19. Fourth, some patients may have developed additional comorbidities or complications since hospital discharge that were not appropriately captured and could potentially affect wellbeing and symptom prevalence and persistence. Fifth, parents/caregivers were interviewed in this study and not the children themselves. There is also a risk of selection bias due to recruitment of the hospitalised population and recall bias in reporting symptoms which were nonexistent at the time of follow-up, and potential selection bias with those with symptoms more likely to agree to the survey.

The study used to generate this data within the ISARIC WHO Clinical Characterisation Protocol initiative is a prospective pandemic preparedness protocol which is agnostic to disease and has a pragmatic design to allow recruitment during pandemic conditions. The reality of conducting research in outbreak conditions does not allow for appropriate co-enrolment of a control group, which is not practical. One of the issues which has not been addressed so far in clinical research is what control group of individuals admitted to hospital during this period when hospitals were overwhelmed with COVID-19 cases could provide a valid control group. The design of this study allows us only to describe the features of COVID-19 survivors and cannot involve a control group. COVID-19 is not just a respiratory tract infection, so there is no "one size fits all” control group. At present, to the best of our knowledge, all major publications on long COVID are uncontrolled cohorts due to the difficulties of ascertaining data among controls matched for age and sex, but most importantly matched for the same experiences during the pandemic aside from confirmed COVID-19 illness.

Our findings have implications for further research. Longer follow-up duration and repeated assessments combined with controls and sampling for further studies into the pathophysiology and immunology of post-COVID-19 illness sequelae are needed to inform case definitions and intervention trials aimed to improve long-term outcomes.

\section{Conclusions}

Although symptoms which were present at discharge diminished over time, even 8 months after hospital discharge many children experienced persistent symptoms, with fatigue, sensory changes and sleep problems being the most common sequelae. One in 10 children experienced multisystem involvement at the time of follow-up. Older age and allergic diseases were the main risk factors for persistent symptoms. Future work should be multidisciplinary, prospective, preferably with a control cohort, repeated sampling, and with an ability for children to report their health and wellbeing themselves, accompanied by biological sample collection to establish causative mechanisms for a better understanding of COVID-19 sequelae and help with the phenotype/endotype categorisation.

Acknowledgements: We are very grateful to the Z.A. Bashlyaeva Children's Municipal Clinical Hospital clinical staff and to the patients, parents, carers and families for their kindness and understanding during these difficult times of the COVID-19 pandemic. We would like to express our very great appreciation to the ISARIC Global COVID-19 Follow-up Working Group for survey development. We would like to thank Maksim Kholopov (Sechenov First Moscow State Medical University (Sechenov University), Moscow, Russia) for providing technical support in data collection and database administration. We are very thankful to Eat \& Talk, Luch, Black Market, FLIP and Academia (Moscow, Russia) for providing us the workspace in a time of need and their support of COVID-19 research. Finally, we would like to extend our gratitude to the ISARIC Global team, the ISARIC Global Adult and Paediatric COVID-19 Follow-up Working Group, and the ISARIC Global Support Centre for their continuous support, expertise and for the development of the outbreak-ready standardised protocols for the data collection.

Sechenov Stop COVID Research Team: Elina Abdeeva, Nikol Alekseeva, Anastasiia Bairashevskaia, Dina Baimukhambetova, Lusine Baziyants, Anna Berbenyuk, Tatiana Bezbabicheva, Julia Chayka, Salima Deunezhewa, Yulia Filippova, Anastasia Gorina, Cyrill Gorlenko, Margarita Kalinina, Bogdan Kirillov, Herman Kiseljow, Natalya Kogut, Mariia Korgunova, Anastasia Kotelnikova, Alexandra Krupina, Anna Kuznetsova, Anastasia Kuznetsova, Veronika Laukhina, Baina Lavginova, Elza Lidjieva, Nadezhda Markina, Daria Nikolaeva, Georgiy Novoselov, Polina Petrova, Erika Porubayeva, Kristina Presnyakova, Anna Pushkareva, Mikhail Rumyantsev, Ilona Sarukhanyan, Jamilya Shatrova, Nataliya Shishkina, Anastasia Shvedova, Valeria Ustyan, Maria Varaksina, Ekaterina Varlamova, 
Margarita Yegiyan and Elena Zuykova (Sechenov First Moscow State Medical University (Sechenov University), Moscow, Russia); Svetlana Gadetskaya and Yulia V. Ivanova (Dept of Paediatrics and Paediatric Infectious Diseases, Institute of Child's Health, Sechenov First Moscow State Medical University (Sechenov University), Moscow, Russia).

Conflict of interest: J. Genuneit reports working as a project manager of unrestricted research grants on the composition of breast milk to Ulm University and Leipzig University with funding from Danone Nutricia Research. M.G. Semple reports grants from the Dept of Health and Social Care National Institute of Health Research UK, grants from the Medical Research Council UK, grants from the Health Protection Research Unit in Emerging \& Zoonotic Infections, University of Liverpool, outside the submitted work; he also reports a minority ownership at Integrum Scientific LLC (Greensboro, NC, USA), outside the submitted work. T. Vos reports personal fees for work on the Global Burden of Disease Study from the Bill and Melinda Gates Foundation, outside the submitted work. C. Apfelbacher has received lecture fees from AstraZeneca, and is a member of a group developing a core outcome set for long COVID, outside the submitted work. All other authors report no relevant conflict of interests.

\section{References}

$1 \quad$ Huang C, Huang L, Wang Y, et al. 6-month consequences of COVID-19 in patients discharged from hospital: a cohort study. Lancet 2021; 397: 220-232.

2 Munblit D, Bobkova P, Spiridonova E, et al. Risk factors for long-term consequences of COVID-19 in hospitalised adults in Moscow using the ISARIC Global follow-up protocol: StopCOVID cohort study. medRxiv 2021; preprint [https://doi.org/10.1101/2021.02.17.21251895].

3 Wise J. Long Covid: WHO calls on countries to offer patients more rehabilitation. BMJ 2021; 372: n405.

4 Editorial. Meeting the challenge of long COVID. Nat Med 2020; 26: 1803.

5 The Lancet. Facing up to long COVID. Lancet 2020; 396: 1861.

6 Office for National Statistics. Statistical bulletin: prevalence of ongoing symptoms following coronavirus (COVID-19) infection in the UK: 1 April 2021. 2021. www.ons.gov.uk/peoplepopulationandcommunity/health andsocialcare/conditionsanddiseases/bulletins/prevalenceofongoingsymptomsfollowingcoronaviruscovid19in fectionintheuk/1april2021 Date last accessed: 6 April 2021.

7 Buonsenso D, Munblit D, De Rose C, et al. Preliminary evidence on long COVID in children. Acta Paediatr 2021; 110: 2208-2211.

8 Say D, Crawford N, McNab S, et al. Post-acute COVID-19 outcomes in children with mild and asymptomatic disease. Lancet Child Adolesc Health 2021; 5: e22-e23.

9 Michelen M, Manoharan L, Elkheir N, et al. Characterising long-term Covid-19: a rapid living systematic review. medRxiv 2020; preprint [https://doi.org/10.1101/2020.12.08.20246025].

10 ISARIC Global COVID-19 Paediatric Follow-Up Working Group. ISARIC Global COVID-19 paediatric follow-up. 2021. https://isaric.org/research/covid-19-clinical-research-resources/paediatric-follow-up Date last accessed: 24 April 2021.

11 Munblit D, Nekliudov NA, Bugaeva P, et al. StopCOVID cohort: an observational study of 3,480 patients admitted to the Sechenov University Hospital Network in Moscow city for suspected COVID-19 infection. Clin Infect Dis 2021; 73: 1-11.

12 Sigfrid L, Buonsenso D, DunnGalvin A, et al. Consequences of COVID-19 infection for child health and wellbeing: protocol for a prospective, observational, longitudinal study in children. 2021. https://osf.io/d2wjn Date last accessed: 24 April 2021.

13 Harris PA, Taylor R, Minor BL, et al. The REDCap consortium: building an international community of software platform partners. J Biomed Inform 2019; 95: 103208.

14 Harris PA, Taylor R, Thielke R, et al. Research electronic data capture (REDCap) - a metadata-driven methodology and workflow process for providing translational research informatics support. $J$ Biomed Inform 2009; 42: 377-381.

15 Davis HE, Assaf GS, McCorkell L, et al. Characterizing long COVID in an international cohort: 7 months of symptoms and their impact. medRxiv 2020; preprint [https://doi.org/10.1101/2020.12.24.20248802].

16 Greenhalgh T, Knight M, A'Court C, et al. Management of post-acute Covid-19 in primary care. BMJ 2020; 370: m3026.

17 Williams K, Thomson D, Seto I, et al. Standard 6: age groups for pediatric trials. Pediatrics 2012; 129: Suppl. 3, S153-S160.

18 Viner R, Gregorowski A, Wine C, et al. Outpatient rehabilitative treatment of chronic fatigue syndrome (CFS/ ME). Arch Dis Child 2004; 89: 615-619.

19 Crawley SA, Caporino NE, Birmaher B, et al. Somatic complaints in anxious youth. Child Psychiatry Hum Dev 2014; 45: 398-407.

20 Sigfrid L, Drake TM, Pauley E, et al. Long Covid in adults discharged from UK hospitals after Covid-19: a prospective, multicentre cohort study using the ISARIC WHO clinical characterisation protocol. medRxiv 2021; preprint [https://doi.org/10.1101/2021.03.18.21253888]. 
21 Taquet M, Geddes JR, Husain M, et al. 6-month neurological and psychiatric outcomes in 236379 survivors of COVID-19: a retrospective cohort study using electronic health records. Lancet Psychiatry 2021; 8: 416-427.

22 Sudre CH, Murray B, Varsavsky T, et al. Attributes and predictors of long COVID. Nat Med 2021; 27: 626-631.

23 Ayoubkhani D, Khunti K, Nafilyan V, et al. Post-Covid syndrome in individuals admitted to hospital with covid-19: retrospective cohort study. BMJ 2021; 372: n693.

24 Afrin LB, Weinstock LB, Molderings GJ. Covid-19 hyperinflammation and post-Covid-19 illness may be rooted in mast cell activation syndrome. Int J Infect Dis 2020; 100: 327-332.

25 Khamsi R. Rogue antibodies could be driving severe COVID-19. Nature 2021; 590: 29-31.

26 Nekliudov NA, Blyuss O, Cheung KY, et al. Excessive media consumption about COVID-19 is associated with increased state anxiety: outcomes of a large online survey in Russia. J Med Internet Res 2020; 22: e20955. 